\title{
Acute Localized Exanthematous Pustulosis (ALEP): Review of Literature with Report of Case Caused by Amoxicillin-Clavulanic Acid
}

\author{
Alessia Villani · Antonello Baldo • Gaia De Fata Salvatores • Vincenzo Desiato • \\ Fabio Ayala · Carlo Donadio
}

Received: September 6, 2017 / Published online: October 19, 2017

(C) The Author(s) 2017. This article is an open access publication

\begin{abstract}
Acute localized exanthematous pustulosis (ALEP) is a localized form of acute generalized exanthematous pustulosis, characterized by acute onset of multiple nonfollicular, pinhead-sized, sterile pustules following drug administration. Antibiotics, especially $\beta$-lactams and macrolides, have been implicated in the majority of cases, although eruption after nonsteroidal antiinflammatory drugs and many other medications has also been reported. Skin reaction arises quickly within a few hours, resolving rapidly within a few days without treatment, and it is usually accompanied by fever and neutrophilic leukocytosis. We report herein all cases of ALEP described in literature, adding the case of a 35-year-old woman admitted to our hospital with outbreak of erythematous pustules on her face, neck, and chest after amoxicillin-clavulanic acid treatment.
\end{abstract}

Enhanced content To view enhanced content for this article go to www.medengine.com/Redeem/ A4CCF0605DBC48AC.

A. Villani $(\bowtie) \cdot$ A. Baldo - G. De Fata Salvatores . V. Desiato - F. Ayala · C. Donadio

Section of Dermatology, Department of Clinical Medicine and Surgery, Dermatology Unit, University of Naples Federico II, Naples, Italy e-mail: ali.vil@hotmail.it
Keywords: AGEP;

ALEP; Amoxicillin-clavulanic acid; Corticosteroids; Exanthematous pustulosis; Hypersensitivity reaction; Sterile pustules

\section{INTRODUCTION}

Acute localized exanthematous pustulosis (ALEP) is an acute skin reaction characterized by acute onset of multiple nonfollicular, pinhead-sized, sterile pustules, developed on an erythematous and edematous background, localized typically to face, neck, or chest $[1,2]$. It is a rare variant of acute generalized exanthematous pustulosis (AGEP), also called toxic pustoloderma [3], both being described histologically as sterile subcorneal pustules rich in neutrophils and clinically characterized by either widespread or localized sterile monomorphic pustules [4]. ALEP can be considered an atypical form of AGEP [1]. The definition of ALEP was introduced in 2005 by Prange et al. [5] to describe a woman who, according to criteria for AGEP, was diagnosed as having localized pustular eruption on the face. Skin reaction arises quickly within a few hours and resolves rapidly within a few days without treatment [6]; moreover, it is usually accompanied by fever and neutrophilic leukocytosis [7]. Although, in $90 \%$ of cases, AGEP and ALEP represent an unusually severe cutaneous hypersensitivity reaction to a systemic drug, 
some cases have also been linked with viral infections and insect bites. Antibiotics, especially $\beta$-lactams and macrolides, are the most frequent causative drugs, although eruption after nonsteroidal antiinflammatory drugs (NSAIDs) and many other medications has also been reported [4, 8]. This review reports all cases of ALEP described in literature, adding the case of a 35-year-old woman admitted to our hospital with outbreak of ALEP after amoxicillin-clavulanic acid treatment.

\section{METHODS}

We searched English-language literature in the PubMed, Embase, Google Scholar, and Scopus databases for publications regarding ALEP through 24 July 2017 using the keywords "acute localized exanthematous pustulosis," "toxic pustoloderma," "toxic pustulosis," "pustulosis," "exanthematous pustulosis," "acute generalized exanthematous pustulosis," "antibiotics," "drug reaction." This article reviews all data regarding this rare form of skin reaction, to provide an update on this disease and its management. Furthermore, we report a new case of ALEP, in a young woman after amoxicillin-clavulanic acid therapy. Informed consent was obtained from the patient for inclusion in the study. All evidence was evaluated by the authors, who then combined this with clinical experience from everyday practice in an effort to provide a complete review of this rare skin reaction.

\section{CASE REPORT}

A 35-year-old woman was admitted to our hospital for acute outbreak of multiple small nonfollicular pustules, single and occasionally with herpetiform disposition, and an underlying erythematous and edematous base, affecting face, neck, and trunk (Fig. 1). There was no mucous membrane, nail, or joint involvement. No itch or sting was referred. At presentation, the patient was febrile (temperature $37.2^{\circ} \mathrm{C}$ ) and presented cervical lymphadenopathy. She had no personal or family history of dermatological diseases. The patient had been taking

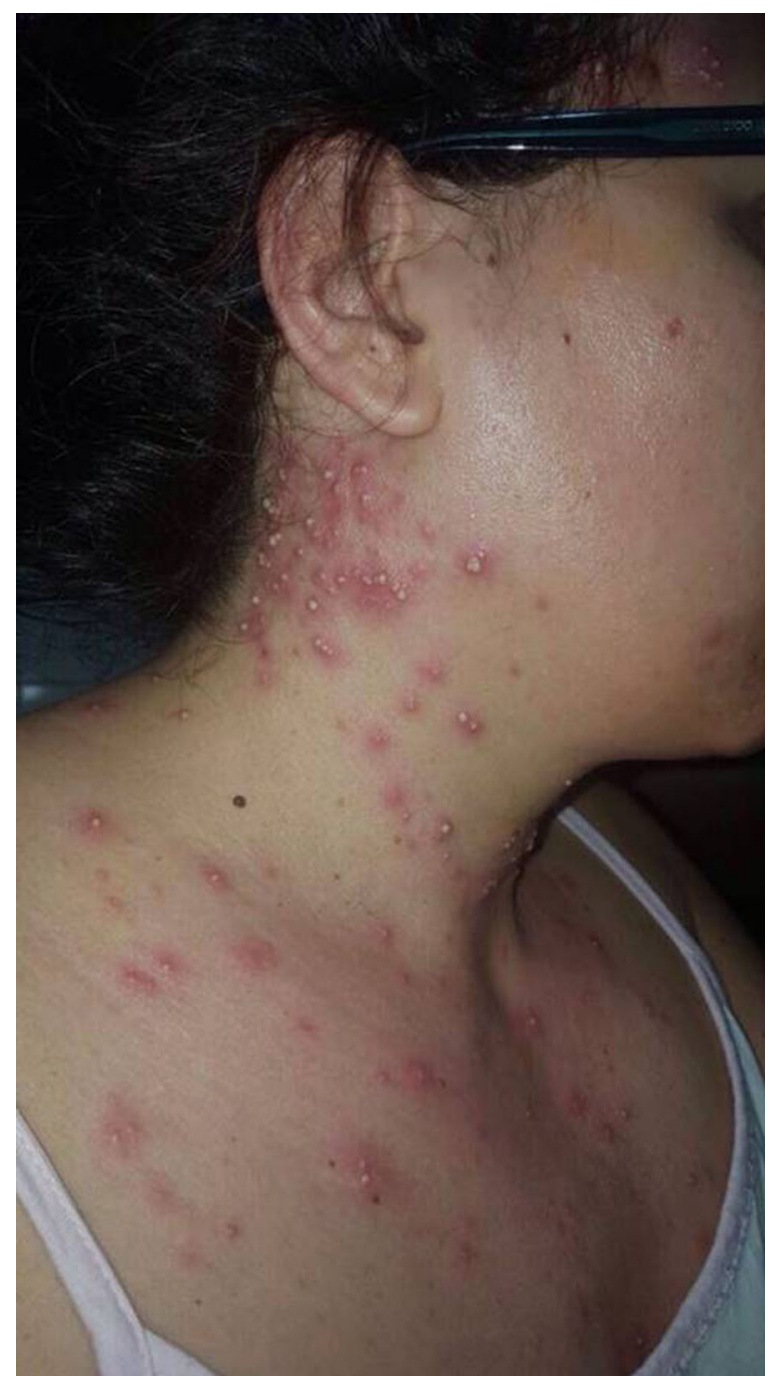

Fig. 1 Multiple erythematous lesions with multiple small nonfollicular pustules localized over neck and trunk

amoxicillin-clavulanic acid ( $1 \mathrm{~g} /$ day for a week) up to 3 days before for pharyngotonsillitis. She took no other medications. Laboratory investigations showed increased white cell count $\left(11,000 / \mathrm{mm}^{3}\right)$ with normal differential. Eosinophilia was not present. Erythrocyte sedimentation rate (ESR, $20 \mathrm{~mm}$ ), C-reactive protein (CRP, $2.6 \mathrm{~g} / \mathrm{L}$ ), and antistreptolysin O (ASO, 376 UI) titer were elevated. Blood chemistry, liver function, and renal function tests were within normal limits. Bacterial and fungal cultures were negative. Results for immunoglobulins [herpes simple virus (HSV)1 and HSV2 immunoglobulin $\mathrm{M}(\operatorname{IgM}) \leq 0.5]$ and viral 
polymerase chain reaction analysis proved absence of herpes simplex virus. Furthermore, skin biopsy from a pustular lesion of the neck was taken. It showed acanthosis of epidermis with mild spongiosis. Moreover, in dermis, there was dense perivascular infiltrate of lymphocytes and neutrophils, resulting in formation of subcorneal, intraepidermal pustules. Patch test for amoxicillin-clavulanic acid was positive. Indeed, use of antibiotics was discontinued and she was treated with systemic corticosteroid (prednisone $37.5 \mathrm{mg}$ daily). The pustules rapidly started to resolve within 5 days after corticosteroid treatment, followed by

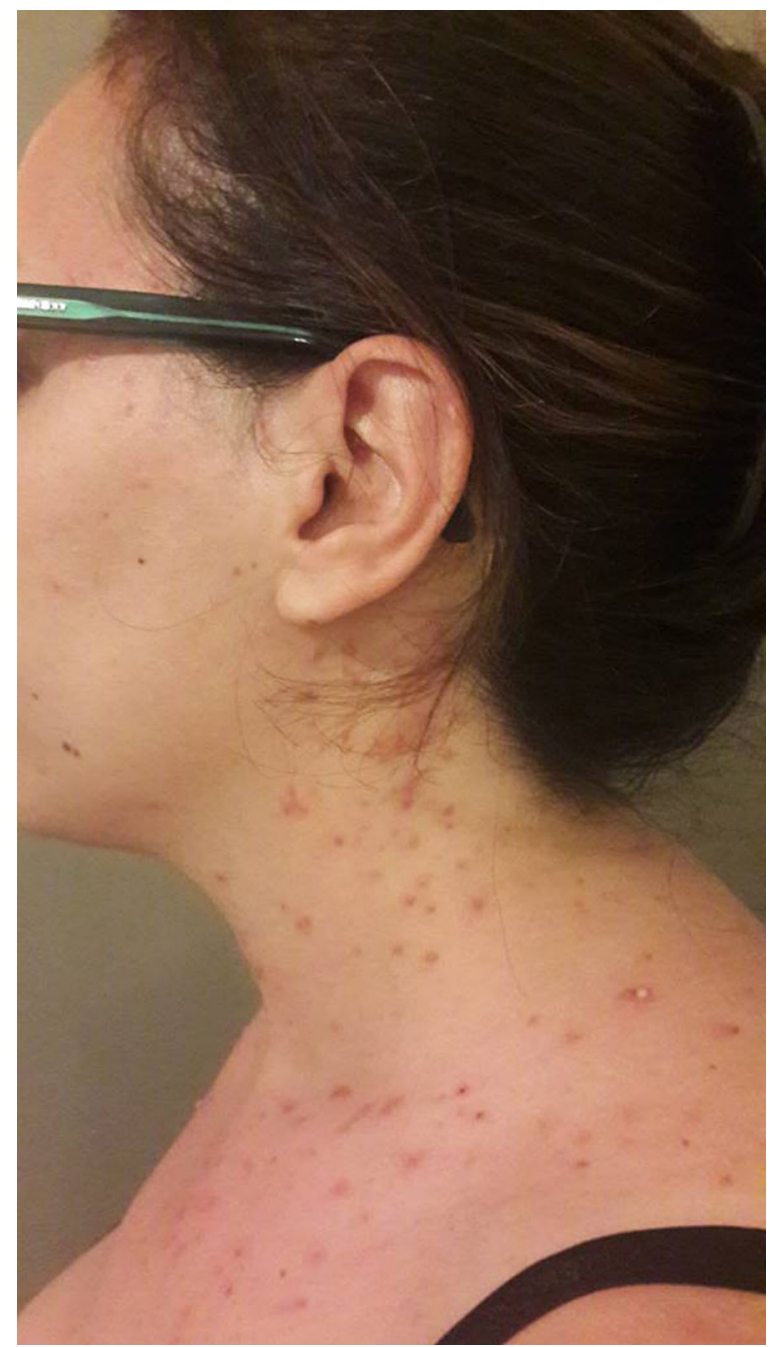

Fig. 2 Within 5 days after withdrawal of amoxicillin-clavulanic acid, the rash resolved slowly, with oral corticosteroid therapy (prednisone $37.5 \mathrm{mg}$ daily) transient residual hyperpigmentation (Fig. 2.). Furthermore, given the temporal relationship between antibiotic administration and skin disease development and its histologic findings, our case can be considered an unusual type of AGEP, defined as ALEP induced by amoxicillin-clavulanic acid.

\section{RESULTS AND DISCUSSION}

Acute localized exanthematous pustulosis occurs only rarely as a particular form of AGEP [1]. About 25 reports have been published in literature (Table 1), confirming that this is a severe cutaneous eruption, which develops abruptly, in the context of recent drug administration, as monomorphic papulopustules with few or no comedones. ALEP characteristically presents with onset of localized 1-2-mm sterile pustules 3-5 days after commencement of a culprit drug, resolving soon after drug withdrawal [9]. Fever and leukocytosis may be present, accompanied by itching or sometimes burning sensation. Mucous membrane involvement is rare, commonly mild, and generally restricted to one site, mostly the oral lips. More than $80 \%$ of cases are drug induced, with antibiotics, especially $\beta$-lactams and macrolides, being the most frequent triggers $[10,11]$, although in particular cases it is induced by bacterial, viral, or parasitic infection $[12,13]$. Although the pathophysiology of both ALEP and AGEP remains unclear, several studies have suggested that AGEP is due to drug-specific T-cell-mediated immune processes (type IVd reaction) [13-15]; in fact, activation, proliferation, and migration of drug-specific cluster of differentiation (CD) 4 and CD8 T cells play an important role in development of the disease, as confirmed by patch test and drug lymphocyte stimulation test (DLST) responses [13]. Clinical differential diagnosis includes follicular eruptions, such as bacterial folliculitis, furunculosis, acne and acneiform pustules, localized pustular contact dermatitis, dermatophyte infection, pyoderma vegetans, varicella, Kaposi varicelliform eruption, Sweet's syndrome, bullous impetigo, impetiginized eczema, pustular erythema multiforme, pemphigus foliaceus and 
Table 1 Published reports on ALEP

\begin{tabular}{|c|c|c|c|c|}
\hline Author(s) & Date & Sex, age & $\operatorname{Drug}(s)$ & Site \\
\hline Shuttleworth & 1989 & - & Amoxicillin & - \\
\hline Jay et al. & 1994 & - & Ampicillin & - \\
\hline Fitzgerald et al. & 1994 & M, 46 & Allopurinol & Face, trunk, limbs \\
\hline $\operatorname{Lim}$ & 1995 & M, 24 & Ampicillin & Face \\
\hline De Argila et al. & 1996 & $\mathrm{~F}, 27$ & Amoxicillin & Trunk \\
\hline Prieto et al. & 1997 & M, 53 & Amoxicillin & Trunk \\
\hline Novalbos et al. & 2000 & - & Ceftibuten and amoxicillin & Face \\
\hline Corbalán-Vélez et al. & 2000 & $\mathrm{~F}, 40$ & Amoxicillin-clavulanic acid & Face, neck, trunk \\
\hline Wohl et al. & 2004 & $\mathrm{~F},-$ & Paracetamol & Neck \\
\hline Prange et al. & 2005 & $\mathrm{~F},-$ & - & Face \\
\hline Corral de la Calle et al. & 2005 & $\mathrm{M}, 70$ & Levofloxacin & Face, neck \\
\hline Betto et al. & 2008 & $\mathrm{~F}, 40$ & Amoxicillin-clavulanic acid & Face \\
\hline Rastogi et al. & 2009 & $\mathrm{~F}, 64$ & Ibuprofen & Face \\
\hline Kim et al. & 2010 & $\mathrm{~F}, 44$ & Docetaxel & Face \\
\hline Liang et al. & 2011 & $\mathrm{~F}, 59$ & Sorafenib & Limbs \\
\hline Ozkaya-Parlakay et al. & 2011 & $\mathrm{~F}, 17$ & Amoxicillin-clavulanic acid & - \\
\hline Sim et al. & 2011 & $\mathrm{~F}, 26$ & $\begin{array}{l}\text { Cephalosporin, } \\
\text { trimethoprim-sulfamethoxazole, } \\
\text { and vancomycin }\end{array}$ & Face \\
\hline Tresch et al. & 2012 & M, 21 & Finasteride & Trunk, abdomen \\
\hline Huilaja et al. & 2014 & M, 44 & Piperacillin-tazobactam & Thighs \\
\hline De Cruz et al. & 2015 & $\mathrm{~F}, 34$ & Clindamycin & Chest \\
\hline Di Meo et al. & 2016 & $\mathrm{~F}, 40$ & Flurbiprofen & Face \\
\hline Qu et al. & 2016 & $\mathrm{~F}, 72$ & Cefoperazone and sulbactam sodium & Face \\
\hline \multirow[t]{2}{*}{ Bala et al. } & 2017 & $\mathrm{~F}, 47$ & Amoxicillin-clavulanic acid & Face, neck, trunk \\
\hline & & $\mathrm{F}, 30$ & & Trunk \\
\hline Our case & 2017 & $\mathrm{~F}, 35$ & Amoxicillin-clavulanic acid & Face, neck, trunk \\
\hline Jha et al. & 2017 & M, 29 & Diclofenac & Trunk \\
\hline
\end{tabular}

other autoimmune bullous disorders, infantile chronic acropustulosis, migratory necrolytic eruption of glucagonoma, bowel bypass syndrome, Behçet's disease, staphylococcal scalded skin syndrome, and others. Most of these can easily be differentiated from AGEP based on clinical appearance, clinical course, and histology, but some diseases whose differentiation from AGEP can cause problems remain [12]. After exposure to the causative agent, antigen-presenting cells present the cognate antigen using major histocompatibility complex (MHC) 
molecules, causing activation of specific CD4 and CD8 cells that proliferate then migrate into dermis and epidermis [13]. Drug-specific CD8 T cells use perforin/granzyme B and Fas ligand mechanisms to induce apoptosis of keratinocytes within epidermis, leading to tissue destruction and epidermal vesicle formation. These cells release increased amounts of C-X-C motif chemokine ligand 8 (CXCL8), a potent neutrophilic cytokine, leading to chemotaxis of neutrophils into vesicles, causing transformation of vesicles into sterile pustules [16, 17]. Furthermore, in vitro tests have shown that Th17 cells may also play a role in the strong neutrophilic activity, by releasing interleukin (IL)-17 and IL-22, which have a synergistic effect on production of CXCL8 and prevention of apoptosis of neutrophils [14]. Some authors have suggested that there is also a genetic component in the development of pustular eruption [18]. Patients with mutations in the IL-36 receptor antagonist (IL36RN) gene are more predisposed to develop AGEP after drug exposure [18]. The IL-36RN gene encodes the IL-36 receptor antagonist, a molecule that blocks inflammatory cytokines such as IL-36 $\alpha$, IL-36 $\beta$, and IL-36 $\gamma$. Therefore, mutations in the IL-36RN gene can result in uncontrolled IL-36 signaling and increased downstream production of IL-6, IL-8, IL- $1 \alpha$, and IL- $1 \beta$ and might predispose to pustular eruptions [12]. Initially, these cases were described as unusual drug eruption, clinically and histologically different from fixed drug eruption [18]. In 1989, Shuttleworth [19] reported the first case of an unusual localized pustular reaction to amoxicillin. The rash was recurrent and always appeared in the same region, but histological findings differed from those of fixed drug eruption. Indeed, the histology of AGEP and ALEP shows spongiform, subcorneal, and/or intraepithelial pustules, edematous papillary dermis, and perivascular infiltrates with neutrophils and some eosinophils. Necrotic keratinocytes and leukocytoclastic vasculitis can also be found [12]. Moreover, our case of ALEP is not the first induced in association with amoxicillin-clavulanic acid treatment; five other cases $[7,15,20-22]$ of amoxicillin-clavulanic acid-induced ALEP have been reported in literature previously (Table 1). Interestingly, all patients were women and the major areas affected by pustular lesions were photoexposed such as face, neck, and chest, simulating acneiform eruption. Recently, Bala et al. reported the cases of two Caucasian women with a diagnosis of amoxicillin-induced localized folliculitis in the spectrum of ALEP [20], based on the clinical presentation of localized monomorphic pustulosis and pathological findings of suppurative folliculocentric acute inflammatory reaction. The time period from drug exposure to reaction onset is typically within $48 \mathrm{~h}$, as reproduced in a double-blind placebo-controlled test in a 53year-old man who developed recurrent localized exanthematous pustulosis at day 7 of 8 days of amoxicillin treatment (500 mg daily) [3]. Since the first description of the disease, the list of drugs involved has grown. There has been only one case showing a relationship between ALEP and levofloxacin [23], in a 70-year-old man treated for pneumonia. The patient presented multiple nonfollicular pinhead-sized pustules over head and neck with fever, which disappeared on interruption of the antimicrobial drug [20]. Other cases of ALEP, including treatment with cefoperazone and sulbactam sodium, piperacillin-tazobactam, and vancomycin, have also been described [1, 11, 24, 25]. Although ALEP is typically localized to photoexposed areas, a particular case reported development of annular erythematous lesions with dozens of tiny nonfollicular pustules on the inguinal area of a 44-year-old man after 1 day of piperacillin-tazobactam therapy for pancreatitis [24]. Although AGEP and psoriasis are the most common vesiculopustular eruptions occurring during pregnancy, two cases of ALEP in pregnant women have been reported. One case described a pustular eruption, localized on the right breast, in a 34-year-old woman who was 19 weeks pregnant after 3 days of treatment with oral clindamycin (300 mg twice daily) [26], while the other case reported acute exanthematous pustulosis localized in the neck region after paracetamol therapy [27]. Other nonantibiotic drugs causing ALEP include NSAIDs (flurbiprofen, ibuprofen, and diclofenac) [28-30], allopurinol [31], and finasteride [32]. Dermoscopy may help in diagnosis at an early stage, as described by Jha et al. in their article; on polarized dermoscopy, 
ALEP appears as small, milky, roundish globules, histologically corresponding to nonfollicular subcorneal pustules, and a pinkish-reddish background, consistent with dermal inflammation [30]. Associations with docetaxel and sorafenib in two patients with carcinoma have also been reported [33, 34]. Finally, information extracted from published case reports in literature shows that, as ALEP is a self-limited disease, the mainstay of treatment is removal of the suspected drug, which leads to improvement in symptoms within several days [35]. Supportive therapy with topical or oral corticosteroids may be appropriate for treatment of pruritus and inflammation in prolonged cases [4].

\section{CONCLUSIONS}

ALEP is an uncommon cutaneous drug reaction characterized by eruption of localized sterile pustules following drug administration. Most published cases involved antibiotics, but many other drugs have been reported to be responsible for this skin reaction. There are no standardized guidelines, and data regarding pathogenesis and treatment of this disease are still unclear, so physicians usually have to rely on published experience from case reports and case series. Indubitably, more studies and international registry are needed to better recognize and treat this disease.

\section{ACKNOWLEDGEMENTS}

No funding or sponsorship was received for this study or publication of this article. All named authors meet the International Committee of Medical Journal Editors (ICMJE) criteria for authorship of this manuscript, take responsibility for the integrity of the work as a whole, and have given final approval for the version to be published.

Disclosures. Alessia Villani, Antonello Baldo, Gaia De Fata Salvatores, Vincenzo Desiato, Fabio Ayala, and Carlo Donadio have nothing to disclose
Compliance with Ethics Guidelines. Informed consent was obtained from the patient for inclusion in the study.

Open Access. This article is distributed under the terms of the Creative Commons Attribution-NonCommercial 4.0 International License (http://creativecommons.org/licenses/ by-nc/4.0/), which permits any noncommercial use, distribution, and reproduction in any medium, provided you give appropriate credit to the original author(s) and the source, provide a link to the Creative Commons license, and indicate if changes were made.

\section{REFERENCES}

1. Sim HS, Seol JE, Chun JS, Seo JK, Lee D, Sung HS. Acute localized exanthematous pustulosis on the face. Ann Dermatol. 2011;23(Suppl 3):S368-70.

2. Mohamed M, Soua Y, Njim L, Hammemi S, Youssef M, Akkari H, Belhadjali H, Zili J. Acute localized exanthematous pustulosis on the face: 6 cases in Tunisia. Ann Dermatol Venereol. 2014;141(12):756-64.

3. Prieto A, de Barrio M, López-Sáez P, Baeza ML, de Benito V, Olalde S. Recurrent localized pustular eruption induced by amoxicillin. Allergy. 1997;52(7):777-8.

4. Roujeau JC, Bioulac-Sage P, Bourseau C, et al. Acute generalized exanthematous pustulosis. Analysis of 63 cases. Arch Dermatol. 1991;127:1333-8.

5. Prange B, Marini A, Kalke A, et al. Acute localized exanthematous pustulosis (ALEP). J Dtsch Dermatol Ges. 2005;3:210-2.

6. de Thier F, Blondeel A, Song M. Acute generalized exanthematous pustulosis induced by amoxycillin with clavulanate. Contact Dermatitis. 2001;44:114-5.

7. Corbalán-Vélez R, Peón G, Ara M, et al. Localized toxic follicular pustuloderma. Int $\mathrm{J}$ Dermatol. 2000;39:209-11.

8. Wolkenstein P, Chosidow O, Fléchet M, et al. Patch testing in severe cutaneous adverse drug reactions, including Stevens-Johnson syndrome and toxic epidermal necrolysis. Contact Dermatitis. 1996;35:234-6. 
9. Vassallo C, Derlino F, Brazzelli V, D'Ospina RD, Borroni G. Acute generalized exanthematous pustulosis: report of five cases and systematic review of clinical and histopathological findings. G Ital Dermatol Venereol. 2014;149:281-90.

10. Novalbos A, Bombín C, Figueredo E, Lluch M, Sastre J. Localized pustulosis induced by betalactams. J Investig Allergol Clin Immunol. 2000;10(3):178-9.

11. Jay S, Kang J, Watcher MA, Broska P, Jeffes EW 3rd. Localized pustular skin eruption. Localized pustular drug eruption secondary to ampicillin. Arch Dermatol. 1994;130(6):787, 790.

12. Szatkowski J, Schwartz RA. Acute generalized exanthematous pustulosis (AGEP): a review and update. J Am Acad Dermatol. 2015;73(5):843-8.

13. Girardi $\mathrm{M}$, Duncan KO, Tigelaar RE, et al. Cross comparison of patch-test and lymphocyte proliferation responses in patients with a history of acute generalized exanthematous pustulosis. Am J Dermatopathol. 2005;27:343-6.

14. Kabashima R, Sugita K, Sawada Y, Hino R, Nakamura M, Tokura Y. Increased circulating Th17 frequencies and serum IL-22 levels in patients with acute generalized exanthematous pustulosis. J Eur Acad Dermatol Venereol. 2011;25(4):485-8.

15. Betto P, Germi L, Bonoldi E, Bertazzoni M. Acute localized exanthematous pustulosis (ALEP) caused by amoxicillin-clavulanic acid. Int $\mathrm{J}$ Dermatol. 2008;47(3):295-6.

16. Lazarov A, Livni E, Halevy S. Generalized pustular drug eruptions: confirmation by in vitro tests. J Eur Acad Dermatol Venereol. 1998;10:36-41.

17. Koga C, Kabashima K, Shiraishi N, Kobayashi M, Tokura Y. Possible pathogenic role of Th17 cells for atopic dermatitis. J Invest Dermatol. 2008; 128:2625-30.

18. Navarini AA, Simpson MA, Borradori L, Yawalkar N, Schlapbach C. Homozygous missense mutation in IL36RN in generalized pustular dermatosis with intraoral involvement compatible with both AGEP and generalized pustular psoriasis. JAMA Dermatol. 2015;151:452-3.

19. Shuttleworth D. A localized, recurrent pustular eruption following amoxycillin administration. Clin Exp Dermatol. 1989;14(5):367-8.

20. Rajgopal Bala H, Jalilian C, Goh MS, Williams R, Tan G, Chong AH. Two cases of amoxycillin-induced follicular acute localised exanthematous pustulosis. Australas J Dermatol. 2017;58(1):e23-5.
21. Ozkaya-Parlakay A, Azkur D, Kara A, Yildiz Y, Orhan $D$, Cengiz AB, Ersoy-Evans S. Localized acute generalized exanthematous pustulosis with amoxicillin and clavulanic acid. Turk J Pediatr. 2011;53(2):229-32.

22. De Argila D, Ortiz-Frutos J, Rodriguez-Peralto JL, Iglesias L. Un caso atípico de pustulosis exantemática aguda generalizada [An atypical case of non-generalized acute exanthematic pustulosis]. Actas Dermo-Sifiliográficas. 1996;87:475-8.

23. de la Calle Corral. M, Martín Díaz MA, Flores CR, Vidaurrazaga C. Acute localized exanthematous pustulosis secondary to levofloxacin. Br J Dermatol. 2005;152(5):1076-7.

24. Huilaja Laura, Kallioinen Matti, Soronen Minna, Riekki Riitta, Tasanen Kaisa. Acute localized exanthematous pustulosis on inguinal area secondary to piperacillin/tazobactam. Acta Derm Venereol. 2014;94:106-7.

25. Qu YJ, Jin SB, Han XC, Zheng LQ. Acute localized exanthematous pustulosis caused by cefoperazone and sodium sulbactam. An Bras Dermatol. 2016;91(6):808-10.

26. De Cruz R, Ferguson J, Wee JS, Akhras V. Acute localised exanthematous pustulosis (ALEP) induced by clindamycin in pregnancy. Australas J Dermatol. 2015;56(3):e55-8.

27. Wohl Y, Goldberg I, Sharazi I, et al. A case of paracetamol induced acute generalized exanthematous pustulosis in a pregnant woman localized in the neck region. SKINmed. 2004;3:47-9.

28. Di Meo N, Stinco G, Patrone P, Trevisini S, Trevisan G. Acute localized exanthematous pustulosis caused by flurbiprofen. Cutis. 2016;98(5):E9-11.

29. Rastogi S, Modi M, Dhawan V. Acute localized exanthematous pustulosis (ALEP) caused by Ibuprofen. A case report. Br J Oral Maxillofac Surg. 2009;47:132-4.

30. Jha AK, Sonthalia S, Lallas A. Non-follicular milky globules-dermoscopy saves the day. Dermatol Pract Concept. 2017;7(2):35-6.

31. Fitzgerald DA, Heagerty AH, Stephens M, Smith AG. Follicular toxic pustuloderma associated with allopurinol. Clin Exp Dermatol. 1994;19(3):243-5.

32. Tresch S, Cozzio A, Kamarashev J, et al. T cell-mediated acute localized exanthematous pustulosis caused by finasteride. J Allergy Clin Immunol. 2012;129:589-94. 
33. Liang CP, Yang CS, Shen JL, Chen YJ. Sorafenib-induced acute localized exanthematous pustulosis in a patient with hepatocellular carcinoma. Br J Dermatol. 2011;165(2):443-5.

34. Kim SW, Lee UH, Jang SJ, Park HS, Kang YS. Acute localized exanthematous pustulosis induced by docetaxel. J Am Acad Dermatol. 2010;63(2):e44-6.

35. Lim JT, Ng SK. An unusual drug eruption to ampicillin. Cutis. 1995;56(3):163-4. 